\title{
Supraciliary Hema Implant in Combined Deep Sclerectomy and Phacoemulsification: One Year Results
}

\author{
Rosa Bonilla*, Jordi Loscos, Xavier Valldeperas, Maria Àngels Parera and Antoni Sabala
}

Department of Ophthalmology, Hospital Universitari Germans Trias i Pujol, Barcelona, Spain

\begin{abstract}
We present the combined surgery of non-penetrating deep sclerectomy with insertion of the implant in the supraciliary space as an effective and safe surgery for patients with both cataract and primary open angle glaucoma. This study included 20 eyes of 16 patients who were followed up during 12 months. We found a significant intraocular pressure reduction, changing from a preoperative mean of $23 \pm 5 \mathrm{mmHg}$ to a postoperative mean of $18 \pm 3 \mathrm{mmHg}$ $(\mathrm{p}<0.002)$. Similarly, a significant reduction in the number of glaucoma drugs needed was observed, varying from $2.5 \pm$ 0.9 drops per patient to $0.7 \pm 0.9(\mathrm{p}<0.0002)$ at the end of the study. We also report a significant improvement in bestcorrected visual acuity, from $5 / 10 \pm 2 / 10$ to $8 / 10 \pm 2 / 10(p<0.006)$, one year after the combined surgery. The only intraoperative complication observed was the microperforation of the trabeculo-descemetic membrane (TDM) and postoperative complications were iris incarceration, seidel test positivity and microhyphema. All these complications resolved successfully.
\end{abstract}

Keywords: Open angle glaucoma, non-penetrating glaucoma surgery, combined surgery, deep sclerectomy, suprachoroidal implant.

\section{INTRODUCTION}

The combination of phacoemulsification and nonpenetrating deep sclerectomy (phaco-NPDS) has been used for the surgical treatment of cataract and open angle glaucoma (OAG) since the first reports in the late 90s $[1,2]$. It has proved its efficacy and safety when compared with other surgical techniques, such as phacoemulsification combined with trabeculectomy $[3,4]$.

Deep sclerectomy is a non-penetrating procedure for the treatment of OAG that can be enhanced with the use of antimetabolites, such as mitomycin C (MMC) or 5fluorouracil, as well as with implants. These devices are placed to facilitate the aqueous outflow, by maintaining the virtual space created after removing the deep scleral flap. There is proof of the good long-term outcomes of its implantation in the scleral bed $[5,6]$, but there is only one report of the placement of the implant in the supraciliary space [7]. The present study is the first report showing the outcomes of deep sclerectomy with supraciliary implant combined with cataract surgery.

\section{MATERIALS AND METHODOLOGY}

Twenty-three eyes of 19 patients with significant cataract and medically uncontrolled $\mathrm{OAG}$, were included in this prospective study. Exclusion criteria were age less than 50 years, African-American race, concurrent ocular inflammatory diseases, previous ocular surgeries, brunescent cataract or angle synechia of $180^{\circ}$ degrees or more. Twenty eyes of 16 patients completed the 12 months follow up and were considered statistical purposes. The other three patients

*Address correspondence to this author at the Hospital Universitari Germans Trias i Pujol, Servicio de Oftalmología, Carretera de Canyet s/n, 08916 Badalona, Spain; Tel: 0034669201633 ;

E-mail: mrosabonilla@gmail.com did not finish the study for several reasons: one patient failed to attend the appointments; another developed an advanced degree of senile dementia during the follow up; and the third patient had to be excluded from the study due to intraoperative microperforation followed by a postoperative irreducible iris incarceration that needed early reintervention changing to a trabeculectomy. We did not consider these three patients in the outcome data, but we included them in safety analyses.

Medically uncontrolled $\mathrm{OAG}$ was defined as an intraocular pressure (IOP) higher than $21 \mathrm{mmHg}$ under a maximal tolerable therapy with evidence of progression of visual field defects and/or optic nerve cupping.

Demographics of the sample are shown in Table 1. In the preoperative assessment, patients underwent a complete examination including best-corrected visual acuity (BCVA) in Snellen decimal scale, biomicroscopy, Goldmann tonometry, gonioscopy, funduscopy. We performed a corneal pakimetry using an ultrasonic pakimeter (UP-1000®, Nidek, Tokyo, Japan) and an ultrasonic immersion biometry for the intraocular lens (IOL) calculation (Ocuscan ${ }^{\circledR}$, Alcon, Fort Worth, TX, USA). All patients also underwent an automated perimetry (Humphrey 740i, Carl Zeiss Meditec, Dublin, CA, USA), staging their visual field damage as mild, moderate or advanced defect according to Hodapp, Parish and Arderson classification [8].

\section{Surgical Technique}

All surgeries were performed, under retrobulbar anaesthesia and intravenous sedation, by the same surgeon (JL). The surgical procedure included a standard phacoemulsification, intraocular lens (IOL) insertion in the capsular bag (Monobloc IOL Y601075, AJL Ophthalmics, Álava, Spain) and a corneal suture. After the cataract surgery, a deep sclerectomy was performed following the 
Table 1. Demographics of the Sample

\begin{tabular}{|c|c|c|c|c|}
\hline & $\mathbf{N}$ & Mean & SD & Range \\
\hline $\begin{array}{ll}\text { Sex } & \\
- & \text { Female } \\
- & \text { Male }\end{array}$ & $\begin{array}{l}5(31.3 \%) \\
11(69.7 \%)\end{array}$ & & & \\
\hline $\begin{array}{ll}\text { Eye } & \\
- & \text { Right } \\
- & \text { Left }\end{array}$ & $\begin{array}{l}8(40 \%) \\
12(60 \%)\end{array}$ & & & \\
\hline \begin{tabular}{ll}
\multicolumn{2}{l}{ Type of glaucoma } \\
- & POAG \\
- & PSXG \\
- & PG
\end{tabular} & $\begin{array}{l}16(80 \%) \\
2(10 \%) \\
2(10 \%)\end{array}$ & & & \\
\hline $\begin{array}{ll}\text { Campimetric stage } \\
\text { - } & \text { Severe } \\
- & \text { Moderate } \\
\text { - } & \text { Not assessable }\end{array}$ & $\begin{array}{l}15(75 \%) \\
3(15 \%) \\
2(10 \%)\end{array}$ & & & \\
\hline Age (years) & & 76.35 & 6.88 & $61-89$ \\
\hline VA (Snellen decimal chart) & & $5 / 10$ & $2 / 10$ & $1 / 10-10 / 10$ \\
\hline IOP (mmHg) & & 22.80 & 4.93 & $16-36$ \\
\hline Number of medications & & 2.55 & 0.94 & $0-4$ \\
\hline Pakimetry $(\mu \mathrm{m})$ & & 556.6 & 44.47 & $490-630$ \\
\hline
\end{tabular}

technique firstly described by Mermoud [9] with the use of MMC. The hema implant (V-2000, Esnoper ${ }^{\circledR}$, AJL Ophthalmics, Álava, Spain) (Fig. 1) was placed in a full thickness scleral pocket, made with a $45^{\circ}$ blade for the incision and a blunt spatula, $2 \mathrm{~mm}$ behind the scleral spur and sutured with a 10/0 nylon suture (Fig. 2).

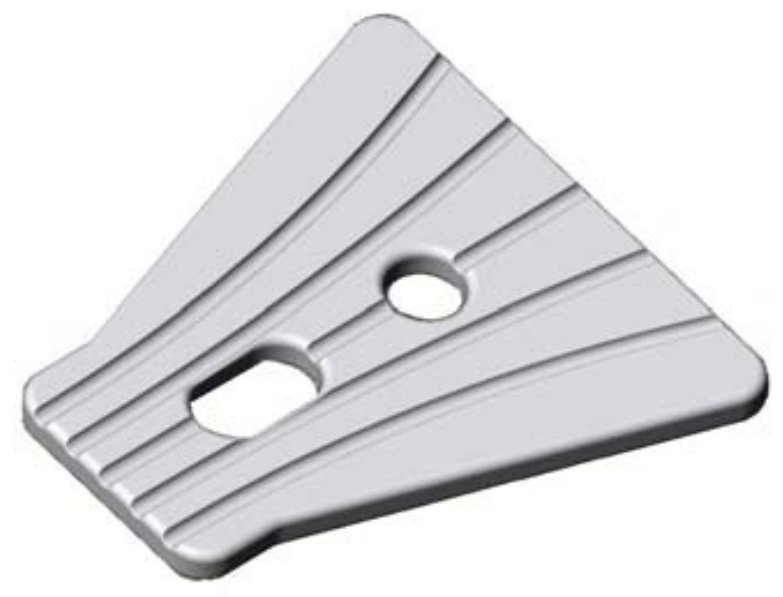

Fig. (1). Hema implant model. (V-2000, Esnoper ${ }^{(B)}$, AJL Ophthalmics, Álava, Spain).

Follow up visits were scheduled at one day, one month, 3 months, 6 months and 12 months after the surgery and all visits included BCVA and IOP measurement. The need for glaucoma treatment or the performance of a goniopuncture were recorded. Topical glaucoma medication was started if the IOP was higher than $21 \mathrm{mmHg}$ or there were signs of glaucoma progression during the follow up. A goniopuncture was considered when IOP was higher than $12 \mathrm{mmHg}$. Only the 12 months results were analysed for statistical purposes.

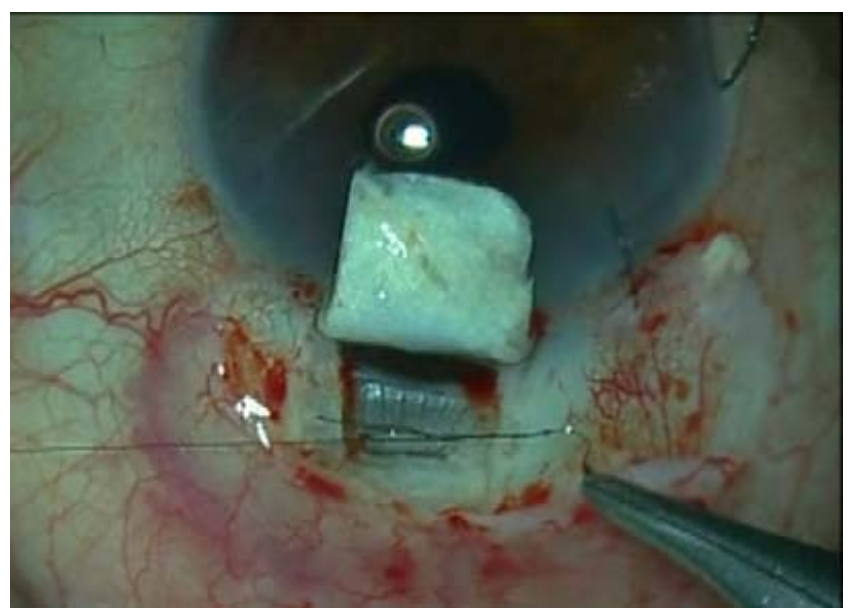

Fig. (2). An intraoperatory image of the implant placement in the supraciliary space while being sutured.

Complete success was defined as IOP less or equal to 21 $\mathrm{mmHg}$ without additional glaucoma medication and qualified success as IOP less or equal to $21 \mathrm{mmHg}$ with or without additional glaucoma medication. The outcome was considered a failure when IOP was lower than $6 \mathrm{mmHg}$ or greater than $21 \mathrm{mmHg}$ despite the use of glaucoma drops, the eye required further glaucoma surgery or lost perception of light [10].

\section{Statistical Analysis}

The quantitative variables were IOP, number of glaucoma drugs used and BCVA, before and after the surgery. This data is presented as mean and standard deviation. We used the d'Agostino-Pearson normality test to assess the symmetry and kurtosis of the variables. The two tails Wilcoxon test for paired data with a $95 \%$ confidence interval was used for the statistical analysis of the sample, and a $p$ value of 0.05 or less was considered as significant.

\section{RESULTS}

Mean preoperative IOP was $22.8 \pm 4.9 \mathrm{mmHg}$ in our sample. Twelve months after the surgery we observed an statistically significant IOP reduction, with a mean of $18 \pm$ $3.1 \mathrm{mmHg}$ after this period $(\mathrm{p}<0.002)$.

There was also an statistically significant reduction in the number of topical treatments after the surgery, starting with a mean of $2.5 \pm 0.9$ drops per patient and decreasing to $0.7 \pm$ 0.9 at the end of the study $(\mathrm{p}<0.0002)$. BCVA improved from $5 / 10 \pm 2 / 10$ to $8 / 10 \pm 2 / 10(p<0.006)$ one year after the surgery. Complete success was achieved in $70 \%$ of the patients and qualified success in $90 \%$ of them, with a failure rate of $10 \%, 6$ months postoperatively. Twelve months after the surgery, complete success was $45 \%$, qualified success was $95 \%$ and the failure rate $5 \%$.

At the end of the study, $80 \%$ of the patients had undergone Nd:Yag goniopuncture, with a mean time between the surgery and the procedure of $77.4 \pm 63.4$ days. No complications related to goniopuncture were recorded. 
For the safety analysis we considered the 23 eyes that underwent surgery. The only intraoperative complication was the microperforation of the trabeculo-descemetic membrane (TDM), observed in four patients. There were no complications related to the phacoemulsification procedure. Postoperative complications included microhyphema in three patients and seidel test positivity (lack of watertightness of the bleb) in two patients 24 hours after the surgery. Both seidel test positivity cases resolved spontaneously one week after the surgery. Iris incarceration in the trabeculodescemetic membrane (TDM) was observed in two patients, and it was successfully managed with green-laser iridoplasty and Nd:Yag-laser lysis of the synechia in one of them. The other case was irreducible and had to be reconverted into a trabeculectomy.

\section{DISCUSSION}

The surgical management of patients with two concurrent ophthalmic pathologies can be controversial. In cases of glaucoma associated with cataract, the approach will depend on the type of glaucoma and its stage, the visual requirements of the patient as well as the surgical experience of the surgeon [11]. The efficacy and safety of phaco-NPDS, a surgical option for these patients, has been shown in several studies $[1,2,6,12-16]$. This surgery can be enhanced with the use of MMC or an implant placement. Both procedures have shown its efficacy to potentiate the IOP reduction $[6,17,18]$. We have used a supraciliary hema implant in order to improve the uveoscleral outflow of aqueous humour through the floor of the implant by maintaining the intraescleral and subconjunctival outflow paths [7]. This three filtering spaces can be visualized on the ultrasound biomicroscopy (Fig. 3).

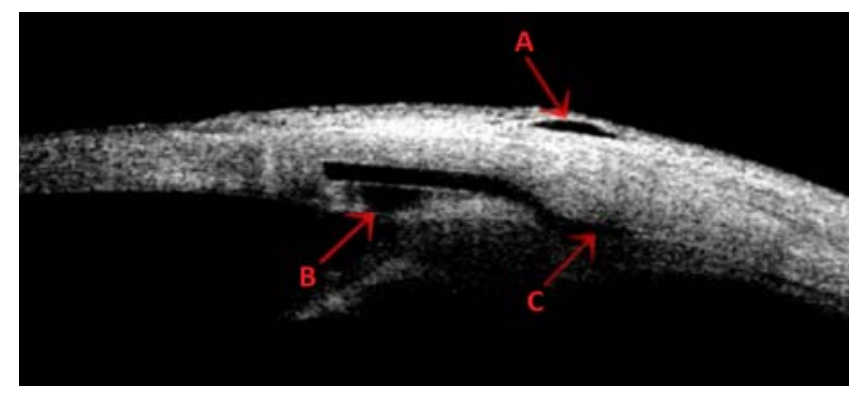

Fig. (3). Ultrasound biomicroscopy of a bleb. The red arrows show the subconjunctival (A), intraescleral (B) and supraciliary (C) spaces for aqueous drainage.

In our study, we have analysed 20 eyes after phacoNPDS and we have found a $21 \%$ reduction in IOP levels, 12 months after the surgery. According to the classical definition in glaucoma surgery, the complete success rate in our sample is $45 \%$ and the qualified success is $95 \%$ at the end of the follow up. Only one patient was considered as a failure for having an IOP of $22 \mathrm{mmHg}$ under maximal topical medication. Other authors have published similar results: Gianoli [1] reports a 59\% complete success rate and Di Staso [2] finds a 73.3\% success rate, not distinguishing between complete and qualified success. Muñoz-Negrete describes a $67.6 \%$ complete success in a study with 39 patients [12], meanwhile Moreno-López reaches an $80 \%$ complete success without the use of any implant, in a study with 15 eyes of 12 patients [15]. None of these authors have used intraoperative MMC in their studies.

There is only one publication showing the results of NPDS using a supraciliary implant [7]. It is difficult to compare our results with this study, because they evaluated NPDS alone without cataract extraction, and the implant used was different to ours (T-flux. IOLTECH Laboratoires, La Rochelle, France). Nevertheless, he reports an overall success rate of $93.4 \%$ similar to the $95 \%$ of our study.

Although we have used the widely known success rate to evaluate our outcomes and compare them to other studies, it is uncertain whether it is a useful tool to validate a glaucoma surgical technique. Well-recognized cohort studies such as the Advanced Glaucoma Intervention Study [19], the Collaborative Initial Glaucoma Treatment Study [20] or the Early Manifest Glaucoma Trial [21] have proven that lowering IOP correlates well with slower visual field defects progression and recommend a lower target IOP in patients with more evolved disease. This is the reason why target IOP should be individualized.

We have found a significant reduction in the number of topical drugs used by our patients, changing from a mean of $2.5 \pm 0.9$ before the surgery to $0.7 \pm 0.9$ at the end of the follow up. These results are similar to that of Gianoli ( $0.6 \pm$ $0.8)$ [1] and higher than the $0.34 \pm 0.6$ of Muñoz-Negrete [12] or the $0.3 \pm 0.2$ described by Muñoz [7].

The rate of Nd:Yag goniopuncture (80\%) in our group is higher than other studies $[22,23]$. This could be because we understand goniopuncture almost as a part of the NPDS procedure, and not as a solution to a postoperative complication. In our hands it enhances aqueous humour drainage and helps to maintain the uveoscleral, intrascleral and subconjunctival spaces created with the surgery.

The most common intraoperative complication was trabeculo-descemetic membrane(TDM) microperforation, occurring in $17.4 \%$ of our patients. This is slightly higher to what has been previously published by Funnell et al. $(15.7 \%)$ and Anand et al. (14.45\%) [14,18]. This situation is related to a higher incidence of postoperative complications and this is why some authors have evaluated these results independently [14].

The limitations of this study are multiple, but are mainly the small sample size and the lack of a control group. The question whether placing an implant in the supraciliary space produces a higher reduction in IOP than in the scleral space, is a question that cannot be answered with our study, and would require a bigger sample size study comparing both techniques.

\section{CONCLUSION}

Phaco-NPDS with supraciliary implant provides a significant reduction both in IOP and glaucoma topical medication, with minor complications that tend to resolve. This combined surgery allows a fast visual rehabilitation and avoids the discomfort that two separate surgeries represent to the patient.

\section{ACKNOWLEDGEMENT}

AJL Ophthalmics has helped to fund this article. 


\section{CONFLICT OF INTEREST}

The authors have no proprietary interest in any of the materials described in this article.

\section{REFERENCES}

[1] Gianoli F, Schnyder CC, Bovey E, Mermoud A. Combined surgery for cataract and glaucoma: phacoemulsification and deep sclerectomy compared with phacoemulsification and trabeculectomy. J Cataract Refract Surg 1999; 25(3): 340-6.

[2] Di Staso S, Taverniti L, Genitti G, et al. Combined phacoemulsification and deep sclerectomy vs phacoemulsification and trabeculectomy. Acta Ophthalmol Scand Suppl 2000; 232: 5960 .

[3] Hondur A, Onol M, Hasanreisoglu B. Nonpenetrating glaucoma surgery: meta-analysis of recent results. J Glaucoma 2008; 17(2): 139-46.

[4] Cheng JW, Cheng SW, Cai JP, Li Y, Wei RL. Systematic overview of the efficacy of nonpenetrating glaucoma surgery in the treatment of open angle glaucoma. Med Sci Monit 2011; 17(7): RA155-63.

[5] Bissig A, Rivier D, Zaninetti M, Shaarawy T, Mermoud A, Roy S. Ten years follow-up after deep sclerectomy with collagen implant. J Glaucoma 2008; 17(8): 680-6.

[6] Shaarawy T, Mermoud A. Deep sclerectomy in one eye $v s$ deep sclerectomy with collagen implant in the contralateral eye of the same patient: long-term follow-up. Eye (Lond) 2005; 19(3): 298302.

[7] Munoz G. Nonstitch suprachoroidal technique for T-flux implantation in deep sclerectomy. J Glaucoma 2009; 18(3): 262-4.

[8] Hodapp E, Parrish RK, Anderson DR. Clinical decisions in glaucoma. St Louis: Mosby 1993.

[9] Mermoud A. Deep sclerectomy: surgical technique. J Fr Ophtalmol 1999; 22(7): 781-6.

[10] Heuer DK, Barton K, Grehn F, Shaarawy TM, Sherwood MB. Consensus on definitions of success. In: Shaarawy TM, Sherwood MB, Grehn F, Eds. Guidelines on Design and Reporting of Surgical Trials. Amsterdam: Kugler 2008; pp. 15-24.

[11] Vizzeri G, Weinreb RN. Cataract surgery and glaucoma. Curr Opin Ophthalmol 2010; 21(1): 20-4.
[12] Muñoz-Negrete FJ, Rebolleda G, Noval S. Non-penetrating deep sclerectomy combined with phacoemulsification. Results and complications. Arch Soc Esp Oftalmol 2003; 78(9): 499-506.

[13] Cillino S, Di Pace F, Casuccio A, et al. Deep sclerectomy versus punch trabeculectomy with or without phacoemulsification: a randomized clinical trial. J Glaucoma 2004; 13(6): 500-6.

[14] Funnell CL, Clowes M, Anand N. Combined cataract and glaucoma surgery with mitomycin $\mathrm{C}$ : phacoemulsificationtrabeculectomy compared to phacoemulsification-deep sclerectomy. Br J Ophthalmol 2005; 89(6): 694-8.

[15] Moreno-Lopez M, Perez-Alvarez MJ. Short- and medium-term intraocular pressure lowering effects of combined phacoemulsification and non-penetrating deep sclerectomy without scleral implant or antifibrotics. Arch Soc Esp Oftalmol 2006; 81(2): 93-100.

[16] D'Eliseo D, Pastena B, Longanesi L, Grisanti F, Negrini V. Comparison of deep sclerectomy with implant and combined glaucoma surgery. Ophthalmologica 2003; 217(3): 208-11.

[17] Wilkins M, Indar A, Wormald R. Intra-operative mitomycin C for glaucoma surgery. Cochrane Database Syst Rev 2005; 19(4): CD002897.

[18] Anand N, Kumar A, Gupta A. Primary phakic deep sclerectomy augmented with mitomycin C: long-term outcomes. J Glaucoma 2011;20(1): 21-7.

[19] The Advanced Glaucoma Intervention Study (AGIS): 7. The relationship between control of intraocular pressure and visual field deterioration. The AGIS Investigators. Am J Ophthalmol 2000; 130(4): 429-40.

[20] Feiner L, Piltz-Seymour JR, Collaborative Initial Glaucoma Treatment Study. Collaborative Initial Glaucoma Treatment Study: a summary of results to date. Curr Opin Ophthalmol 2003; 14(2): 106-111.

[21] Heijl A, Leske MC, Bengtsson B, et al. Reduction of intraocular pressure and glaucoma progression: results from the Early Manifest Glaucoma Trial. Arch Ophthalmol 2002; 120(10): 1268-79.

[22] Anand N, Pilling R. Nd:YAG laser goniopuncture after deep sclerectomy: outcomes. Acta Ophthalmol 2010; 88(1): 110-5.

[23] Mermoud A, Karlen ME, Schnyder CC, et al. Nd:Yag goniopuncture after deep sclerectomy with collagen implant. Ophthalmic Surg Lasers 1999; 30(2): 120-5.

(C) Bonilla et al.; Licensee Bentham Open.

This is an open access article licensed under the terms of the Creative Commons Attribution Non-Commercial License (http://creativecommons.org/licenses/by$\mathrm{nc} / 3.0 /$ ) which permits unrestricted, non-commercial use, distribution and reproduction in any medium, provided the work is properly cited. 